\title{
As competências para atuação na fronteira do conhecimento entre a engenharia de software e as ciências sociais: um ensaio teórico preliminar
}

\author{
José Jorge Lima Dias Jr., José Adson Oliveira Guedes da Cunha ${ }^{2}$
}

Centro de Ciências Sociais Aplicadas - Universidade Federal da Paraíba (UFPB) João Pessoa - PB - Brasil

${ }^{2}$ Centro de Ciências Aplicadas e Educação - Universidade Federal da Paraíba (UFPB) Rio Tinto - PB - Brasil.

jorge@dcx.ufpb.br, adson@dcx.ufpb.br

Abstract. This paper presents an initial theoretical essay aiming to generate reflections on the challenges related to the studies on social and human aspects in Software Engineering (SE). The focus of the discussion is based on the limitations that the SE community has in working on the frontier of knowledge with other areas and how it affects the generation of scientific knowledge. Hence, an epistemological, methodological and theoretical competency model was proposed as a possible solution.

Resumo. Este artigo apresenta um ensaio teórico inicial com o objetivo de gerar reflexões acerca dos desafios relacionados aos estudos sobre aspectos sociais e humanos na Engenharia de Software (ES). O foco da discussão baseia-se nas limitações que a comunidade de ES possui em trabalhar na fronteira do conhecimento com outras áreas e como isso afeta a geração do conhecimento científico. Desse modo, um modelo de competências epistemológicas, metodológicas e teóricas é proposto como um possível encaminhamento.

\section{Introdução}

A comunidade científica em Engenharia de Software (ES) tem reconhecido a importância das questões sociais, cognitivas, culturais, políticas e organizacionais. Como consequência, cada vez mais são aplicadosos conceitos já consolidados da psicologia e das ciências sociais ao contexto de software [Lenberg et al. 2015]. Nesse sentido, a principal tese defendida neste ensaio preliminar é que há uma necessidade sine qua non de que os pesquisadores de ES caminhem para a fronteira do conhecimento para compreender os fenômenos oriundos da área e assim gerar avanços significativos e relevantes.

A discussão deste artigo concentra-se nos desafios que um pesquisador da área de ES enfrenta ao se deparar com fenômenos de natureza social e/ou humana, já que normalmente sua formação (graduação e pós-graduação stricto sensu) está dentro de uma área considerada de exatas como Ciência da Computação ou equivalente. Desse modo, levantamos o seguinte questionamento: quais competências devem ser desenvolvidas para que esses pesquisadores tenham de fato uma inserção em um campo que toca a fronteira do conhecimento com outras áreas ditas sociais e/ou humanas? 
A contribuição deste artigo, portanto, não está vinculada ao rigor metodológico, como acontece normalmente em estudos da área, mas na capacidade de gerar um esforço reflexivo para compreender a realidade. O objetivo é que as reflexões contidas nesse ensaio fomentem a discussão em torno da tese principal, oportunizando a elaboração de ideias e o encaminhamento de possíveis soluções. Essa discussão é especialmente relevante para os programas de pós-graduação stricto sensu que possuem linhas de pesquisa interdisciplinar entre a ES e as ciências sociais.

Para atender ao objetivo proposto, o artigo segue a seguinte estrutura: a Seção 2 apresenta as barreiras para se chegar à fronteira do conhecimento; a Seção 3 discute as competências que precisam ser desenvolvidas para romper a barreira do conhecimento; e, por fim, a Seção 4 apresenta as considerações finais e trabalhos futuros.

\section{As barreiras para se chegar à fronteira do conhecimento}

Para analisar a barreira que existe para se acessar a fronteira do conhecimento, nos apoiaremos a uma perspectiva sociológica, especificamente através da lente das comunidades de prática [Wenger 1998]. As comunidades de prática são um conjunto de relacionamentos entre pessoas e suas atividades ao longo do tempo, e são definidas pelos seus membros na forma pelas quais certas coisas são feitas e interpretadas por eles [Bispo 2013]. Dessa forma, podemos perceber o ensino e a pesquisa como uma prática social, cujo os membros são os pesquisadores da área.

A Figura 1 ilustra a ideia central deste artigo. Pesquisadores da área de Ciência da Computação estão cada vez mais buscando compreender fenômenos associados ao seu campo empírico. Muitos desses fenômenos, no entanto, tocam a fronteira com outras ciências já bem estabelecidas. Na figura, cada um dos círculos representa uma comunidade de prática. Desse modo, para acessar o fenômeno, é preciso romper a barreira da fronteira do conhecimento, acessando uma área de interseção com outra área da ciência, ou seja, uma outra comunidade de prática.

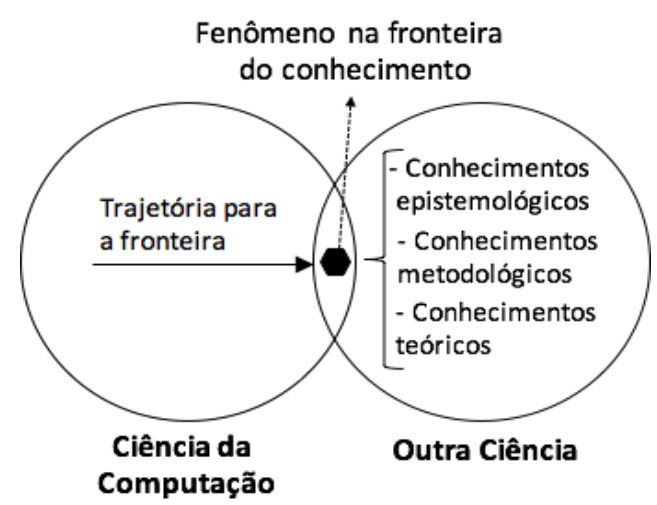

Figura 1. Trajetória de desenvolvimento de competências para atuar na fronteira do conhecimento

O conceito de comunidade de prática está diretamente relacionado com a ideia de participação periférica legitimada [Lave e Wenger 1991], que representa a inserção gradual de um novo membro na comunidade, o qual precisa se apropriar do currículo situado pertencente à comunidade. Neste sentido, a legitimação de um pesquisador de fora da comunidade requer uma adaptação, pois ele precisa entender as práticas, a linguagem, a forma de pensamento e de ação de quem faz parte dela. 
Desse modo, há a necessidade emergente de se apropriar de algumas competências oriundas dessa ciência outra, permitindo que a geração do conhecimento seja de fato substantiva e que não haja uma duplicação ou repetição de descobertas sobre o fenômeno já bem estabelecidas. Conforme sugerido por Lenberg et al. (2015), é necessário considerar os avanços dessas outras ciências para que não fiquemos "reinventando a roda". Araujo et al. (2015) destacam que esta mudança de perspectiva não é trivial para uma comunidade formada por egressos de pesquisa em uma área fim, como a Computação, e que há a necessidade de se trabalhar fortemente não só na comunidade atual, como também no âmbito da formação de novos mestres, doutores e pesquisadores que irão compor a área futuramente.

Esse artigo, escrito na forma de um ensaio teórico, já pode ser considerado como um esforço em direção à fronteira de interseção com as ciências sociais, pois trata-se de um gênero textual comum nessa área, mas que pode gerar inquietações em áreas como a Computação. No entanto, acreditamos na mutabilidade das comunidades de prática, especialmente a ES, que vem buscando cada vez mais ampliar sua perspectiva em compreender a realidade e em fazer ciência.

\section{As competências para romper as barreiras da fronteira do conhecimento}

Conforme apresentado na Figura 1, para se chegar à fronteira do conhecimento é necessário romper uma barreira de uma outra comunidade científica, e se apropriar de um conjunto de conhecimento oriundo dela. A sugestão trazida aqui é o desenvolvimento de algumas competências, organizadas em três eixos: competências epistemológicas, metodológicas e teóricas.

\subsection{Competências epistemológicas}

As abordagens metodológicas, tanto qualitativas quanto quantitativas, fundamentam-se em pressupostos filosóficos que representam "como" o pesquisador irá aprender e "o que" ele irá aprender com a pesquisa. A dimensão epistemológica relaciona-se ao conhecimento e como ele pode ser obtido [Hirschheim 1992]. Os pressupostos filosóficos são classificados de diferentes formas por vários pesquisadores [Burrel e Morgan 1979; Gephart 2004]. No âmbito da computação, no entanto, há um domínio hegemônico do paradigma funcionalista e uma epistemologia positivista.

A perspectiva epistemológica positivista é fortemente caracterizada pela visão determinista, racional e cartesiana sobre os fatos da realidade, com destaque para a separação excludente entre pesquisador e o objeto de pesquisa [Myers 2005]. O interpretativismo, por sua vez, tem o objetivo de entender o mundo do ponto de vista daqueles que o vivenciam. Nessa abordagem, o objeto de pesquisa é entendido como construído socialmente pelos atores [Schwandt 1994].

Apesar dos esforços para definição de padrões, processos e métodos para o desenvolvimento de software, há de se reconhecer que não estamos lidando com uma ciência exata com leis ou regras pré-estabelecidas, mas com um conjunto complexo de tarefas baseadas nas relações humanas e no conhecimento, experiência, caráter e formação cultural de cada indivíduo. Nesse sentido, apesar de a maior parte das pesquisas na área de computação, e, especificamente, na ES, se concentrar na perspectiva positivista, é importante que os pesquisadores abram seus leques para 
investir em métodos que considerem a complexidade dos fatores humanos e das relações sociais no desenvolvimento de software.

\subsection{Competências metodológicas}

A necessidade de se focar no paradigma funcionalista exige a aplicação de métodos de pesquisa usados de forma mais abrangente em outras áreas. A GroundedTheory (GT) provou ser uma abordagem de pesquisa útil em vários campos de pesquisa. Por ser um método baseado em um paradigma indutivo diferente do modelo tradicional de pesquisa hipotético-dedutiva na área de computação, seu uso nem sempre é feito de forma adequada. De acordo com uma revisão sistemática da literatura conduzida por Stol et al. (2016), de 98 artigos que mencionam o uso de GT na engenharia de software, apenas 16 fornecem relatos detalhados de seus procedimentos de pesquisa.

O Estudo de Caso, apesar de utilizado há mais tempo na área de computação, nem sempre o é de acordo com a definição clássica de um método empírico com o objetivo de investigar um fenômeno contemporâneo em seu contexto [Runeson 2009]. Pesquisas na área de melhoria de processos e transferência de tecnologia indicam o uso de Estudo de Caso quando deveriam ser Pesquisa-Ação. O primeiro é puramente observacional enquanto o segundo é focado na mudança do processo.

Diante deste cenário, faz-se necessário que os estudantes tenham acesso a pesquisas em outras áreas relacionadas com o fenômeno para entender como os métodos de pesquisa foram utilizados, dada a maior maturidade no uso de tais métodos nas ciências sociais.

\subsection{Competências teóricas}

A utilidade de uma teoria se dá na organização de um fenômeno tendo em vista a previsão de novos fatos e relacionamentos baseados em outros previamente conhecidos, além de indicar pontos que não foram, de forma convincente, explicados [Marconi e Lakatos 2010].

Em programas de Pós-Graduação em computação é esperado que o foco dos conteúdos das disciplinas seja voltado apenas aos aspectos específicos da área. No entanto, algumas disciplinas específicas a uma linha de pesquisa voltada aos aspectos sociais na ES poderiam ser ofertadas. Uma outra alternativa seria permitir que o aluno curse disciplinas em programas de outras áreas do conhecimento.

Ao se pesquisar fenômenos na fronteira do conhecimento, os pesquisadores precisam consultar trabalhos científicos de outra área. Muitas vezes, o completo entendimento das teorias estudadas ou geradas por esses trabalhos dependem do conhecimento sobre epistemologia e metodologia do pesquisador. Se há uma intenção, por exemplo, em se pesquisar aprendizagem organizacional, é necessário absorver conhecimentos de teorias oriundas da Educação, Administração e/ou Psicologia.

\section{Considerações finais e Trabalhos futuros}

Um dos principais desafios no campo da educação em ES é a diversidade de perfís e enfoques dos programas de pós-graduação na área de Ciência da Computação. Isso sinaliza a dificuldade de criação de modelos de formação voltados especificamente a interseção entre ES e as Ciências Sociais. Esse obstáculo acaba gerando dificuldades no 
desenvolvimento de competências para pesquisadores que desejam atuar na fronteira do conhecimento da computação com outras áreas, como a Psicologia e Administração. Isso se torna um ciclo vicioso, pois os novos professores e pesquisadores serão aqueles que formarão os futuros alunos.

Como trabalho futuro, os autores vislumbram duas ações: (1) analisar as estruturas curriculares de programas de Pós-Graduação na área de Ciência da Computação para identificar gaps nas três dimensões propostas do modelo de competências; e (2) mapear as interseções entre periódicos da Ciência da Computação e periódicos das Ciências Sociais como Psicologia e Administração.

\section{Referências}

Stol, Klaas-Jan; Ralph, Paul; Fitzgerald, Brian. (2016) "Grounded theory in software engineering research: a critical review and guidelines". In: Software Engineering (ICSE), 2016 IEEE/ACM 38th International Conference on. IEEE, 2016. p. 120-131.

Lenberg, P.; Feldt, R.; Wallgren, L. G.. (2015) "Behavioral software engineering: A definition and systematic literature review". Journalof Systems and software, v. 107, p. 15-37.

Wenger, E. (1988), Communities of Practice: learning, meaning and identity. New York: Cambridge.

Lave, J.; Wenger, E. (1991), Situated learning: legitimate peripheral participation. Cambridge: Cambridge University Press.

Bispo, M.(2013) "Aprendizagem organizacional baseada no conceito de prática: contribuições de silviagherardi". Revista de Administração Mackenzie, v.14, n.6, p.132.

Araújo, R. et al. (2005) "A Comunidade de Pesquisa em Sistemas de Informação no Brasil na perspectiva do Simpósio Brasileiro de Sistemas de Informação". iSysRevista Brasileira de Sistemas de Informação, v. 8, n. 1, p. 5-17.

Hirschheim, R. (1992) "Information Systems Epistemology: An Historical Perspective," in Information Systems Research: Issues, Methods and Practical Guidelines, R. Galliers (ed.), Blackwell Scientific Publications, Oxford, pp. 28-60.

Burrel, G.; Morgan, G. (1979), Sociological paradigms and organizational analysis. London: Heinemann Educational Books.

Gephart Jr., R. P. (2004) "Qualitative Research and the Academy of Management Journal".From the Editors. Academy of Management Journal, Vol.47, No.4,454-462.

Myers, M. D. (1997) "Qualitative Research in Information Systems," MIS Quarterly (21:2), June 1997, pp. 241-242. MISQ Discovery.

Schwandt, T. A. (1994), "Constructivist, Interpretivist Approaches to Human Inquiry". In: Denzin, Norman K. Handbook of qualitative research. Thousand Oaks: Sage.

Runeson, P.; Host, M. (2009) "Guidelines for conducting and reporting case study research in software engineering". Empirical software engineering, v.14, n. 2, p. 131.

Marconi, M. A.; Lakatos, E. M. (2010), Fundamentos de Metodologia Científica, 7 Edição, São Paulo: Editora Atlas. 